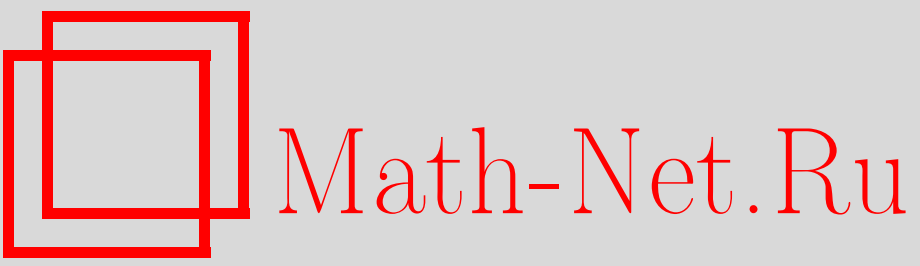

О. Н. Карпенков, О двумерных цепных дробях целочисленных гиперболических матриц с небольшой нормой, УМH, 2004, том 59, выпуск 5, 149-150

DOI: https://doi.org/10.4213/rm778

Использование Общероссийского математического портала Math-Net.Ru подразумевает, что вы прочитали и согласны с пользовательским соглашением

http://www.mathnet.ru/rus/agreement

Параметры загрузки:

IP : 54.196.121.252

26 апреля 2023 г., 17:34:23 


\title{
О ДВУМЕРНЫХ ЦЕПНЫХ ДРОБЯХ ЦЕЛОЧИСЛЕННЫХ ГИПЕРБОЛИЧЕСКИХ МАТРИЦ С НЕБОЛЬШОЙ НОРМОЙ
}

\author{
О. Н. КАРПЕНКОВ
}

Назовем две цепные $(k-1)$-мерные дроби (см. [1]) әквивалентными, если существует линейная замена координат пространства $\mathbb{R}^{k}$, сохраняющая целочисленную решетку, которая переводит одну цепную дробь в другую. В этой заметке мы приводим классификацию двумерных цепных дробей кубических иррациональностей, построенных по матрицам с нормой меньше семи, с точностью до описанного отношения эквивалентности многомерных цепных дробей, опираясь на свойства семейства матриц фробениусова типа. Под нормой матрицы здесь понимается сумма модулей ее коэффициентов. В заключение строится пример матрицы не фробениусова типа. Автор благодарен В.И. Арнольду за постоянное внимание к этой работе и полезные замечания.

Обозначим через $M(k, \mathbb{Z})$ множество $(k \times k)$-матриц с целыми коэффициентами, характеристические многочлены которых неприводимы над полем $\mathbb{Q}$. Отметим, что не существует матриц множества $M(3, \mathbb{Z})$ с нормой меньше трех, а количество матриц множества $M(3, \mathbb{Z})$ с нормой четыре, пять и шесть равно, соответственно, 240, 1248 и 8112.

Пусть $H(k, \mathbb{Z})$ - подмножество матриц из $M(k, \mathbb{Z})$, по которым возможно построить многомерные цепные дроби, т.е. $H(k, \mathbb{Z})$ - подмножество гиперболических матриц, все собственные значения которых вещественны и различны. Количество матриц множества $H(3, \mathbb{Z})$ с нормой меньше четырех, пять и шесть равно соответственно 0,48 и 912.

Фробениусовой матрицей $M_{a_{1}}, \ldots, a_{k}$ назьвается $(k \times k)$-матрица, полученная из единичной матрицы размера $(k-1) \times(k-1)$ дописыванием слева столбика из нулей, а затем снизу строки чисел $\left(a_{k}, \ldots, a_{1}\right)$. Отметим, что характеристический многочлен такой матрицы равен $\chi(x)=$ $(-1)^{k}\left(x^{k}-a_{1} x^{k-1}-\cdots-a_{k-1} x-a_{k}\right)$. Кроме того, матрица $M_{a_{1}, \ldots, a_{k}}$ совпадает с матрицей умножения на элемент $x$ в поле $\mathbb{Q}[x] /(\chi(x))$.

Назовем матрицу множества $H(k, \mathbb{Z})$ матрицей фробениусова типа, если ее многомерная цепная дробь эквивалентна некоторой многомерной цепной дроби, построенной по какой-либо фробениусовой матрице с целыми коэффициентами. Или, что эквивалентно, матрица $A$ из $H(k, \mathbb{Z})$

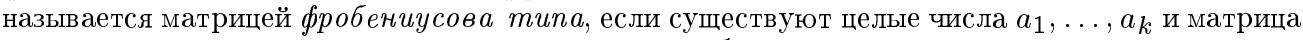
$X$ из группы $S L(k, \mathbb{Z})$, при которых матрица $X A X^{-1}$ коммутирует с $M_{a_{1}}, \ldots, a_{k}$. Это определение естественно обобщается на матрицы множества $M(k, \mathbb{Z})$ : нужно заменить $H(k, \mathbb{Z})$ на $M(k, \mathbb{Z})$.

Теорема 1. Все матрицы множества $M(3, \mathbb{Z})$, норма которых не превосходит шести, являются матрицами фробениусова типа.

Теорема 1 существенно облегчает классификацию двумерных цепных дробей кубических иррациональностей, построенных по матрицам с нормой, не превосходящей шести.

Итак, в множестве $H(3, \mathbb{Z})$ матриц с нормой меньше пяти нет. Если норма равна пяти, то двумерная цепная дробь, построенная по такой матрице, эквивалентна цепной дроби, построенной по матрице $M_{-1,2,1}$. Такая дробь называется золотым сечением. Триангуляция тора (гомеоморфньй тип) этой двумерной цепной дроби для паруса, содержащего точку $(0,0,1):$ строена в работе Е. И. Коркиной (см. [2]). Если норма матрицы равна шести, то возможны только следующие три варианта: соответствующая цепная дробь эквивалентна либо золотому сечению (для 480 матриц); либо цепной дроби матрицы $M_{-1,3,1}:$ (для 240 матриц); либо цепной

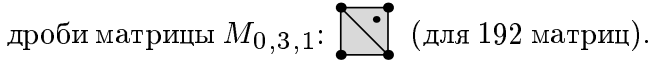

Приведенная классификация, а также доказательство теоремы 1 опирается на обобщение следующей теоремп.

Tеорема 2. Матрица $A=\left(a_{i j}\right)$ из $M(2, \mathbb{Z})$, где $1 \leqslant i, j \leqslant 2$, является матрицей фробениусова типа тогда и только тогда, когда существует челочисленное реше-

Работа выполнена при поддержке грантов INTAS-00-0259, НШ-1972.2003.1 и РФФИ-01-0100660 . 
ние у следующего уравнения: $\left|Q_{A}(x, y)\right|=1$, где $Q_{A}(x, y)=\left(a_{12} x^{2}+\left(a_{22}-a_{11}\right) x y-\right.$ $\left.a_{21} y^{2}\right)\left(\text { НОД }\left(a_{12}, a_{21}, a_{22}-a_{11}\right)\right)^{-1}$.

Через $\Xi(C)$ обозначим множество целочисленных матриц, коммутирующих с $C$. Множество матриц $\bar{\Xi}(C)$, где матрица $C$ из множества $M(k, \mathbb{Z})$, является группой по сложению, изоморфной $\mathbb{Z}^{k}$.

Пусть единичная матрица $E$ и целочисленные матрицы $A$ и $B$ образуют базис группы $\bar{\Xi}(C)$, где $A=\left(a_{i j}\right), 1 \leqslant i, j \leqslant 3$. Поскольку $B$ входит в группу $\Xi(C), B=\alpha A^{2}+\beta A+\gamma E$, где коэффициенты многочлена $\alpha, \beta$ и $\gamma-$ рациональные числа. Пусть характеристический многочлен матрицы $A$ равен $\chi(x)=-x^{3}+a_{1} x^{2}-a_{2} x+a_{3}$. Положим по определению

$$
\begin{aligned}
\bar{P}(m, n)= & m^{3}+\left(2 a_{1} \alpha+3 \beta\right) m^{2} n+\left(\left(a_{2}+a_{1}^{2}\right) \alpha^{2}+4 a_{1} \alpha \beta+3 \beta^{2}\right) m n^{2} \\
& +\left(\left(a_{1} a_{2}-a_{3}\right) \alpha^{3}+\left(a_{2}+a_{1}^{2}\right) \alpha^{2} \beta+2 a_{1} \alpha \beta^{2}+\beta^{3}\right) n^{3} .
\end{aligned}
$$

Для матриц $A=\left(a_{i j}\right)$ и $B=\left(b_{i j}\right)$ положим по определению $\langle i j, k l\rangle_{A, B}=a_{i j} b_{k l}-a_{k l} b_{i j}$ (все индексы пробегают целые числа от одного до трех). Зададим следующий многочлен:

$$
\begin{aligned}
\widetilde{P}_{A, B}(x, y, z)= & \langle 12,13\rangle_{A, B} x^{3}+\langle 23,21\rangle_{A, B} y^{3}+\langle 31,32\rangle_{A, B} z^{3} \\
& +\left(\langle 13,11\rangle_{A, B}+\langle 22,13\rangle_{A, B}+\langle 12,23\rangle_{A, B}\right) x^{2} y \\
& +\left(\langle 22,23\rangle_{A, B}+\langle 23,11\rangle_{A, B}+\langle 13,21\rangle_{A, B}\right) x y^{2} \\
& +\left(\langle 33,12\rangle_{A, B}+\langle 12,11\rangle_{A, B}+\langle 32,13\rangle_{A, B}\right) x^{2} z \\
& +\left(\langle 32,33\rangle_{A, B}+\langle 11,32\rangle_{A, B}+\langle 31,12\rangle_{A, B}\right) x z^{2} \\
& +\left(\langle 21,22\rangle_{A, B}+\langle 33,21\rangle_{A, B}+\langle 23,31\rangle_{A, B}\right) y^{2} z \\
& +\left(\langle 31,22\rangle_{A, B}+\langle 33,31\rangle_{A, B}+\langle 21,32\rangle_{A, B}\right) y z^{2} \\
& +\left(\langle 11,22\rangle_{A, B}+\langle 22,33\rangle_{A, B}+\langle 33,11\rangle_{A, B}+3\langle 13,31\rangle_{A, B}\right) x y z .
\end{aligned}
$$

Teорема 3. Матрица $C$ из $M(3, \mathbb{Z})$ является матрищей фробениусова типа тогда и только тогда, когда существует челочисленное решение следующего уравнения $c$ чельми коэффициентами на неизвестные $x, y, z, m u n:\left|Q_{A, B}(x, y, z ; m, n)\right|=1$, где

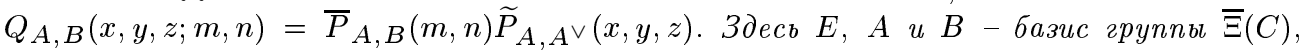
а через $A^{\vee}$ обозначена матрица, на $(\mathrm{ij})$-м месте которой стоит дополнительный минор, умно женный на $(-1)^{i+j}$

СлЕДСТВИЕ ТЕОРЕмы 3. Следующая матрица не фробениусова типа:

$$
A=\left(\begin{array}{ccc}
1 & 2 & 0 \\
0 & 1 & 2 \\
-7 & 0 & 29
\end{array}\right)
$$

ДокАЗАТЕЛЬСтво. Для базиса $E, A$ и $B=\frac{A^{2}}{2}-15 A+\frac{29 E}{2}$ класса $\bar{\Xi}(A)$ уравнение $\left|Q_{A, B}\right|=$ 1 не разрешимо в целых числах, поскольку оно не разрешимо по модулю семь. Следовательно, по теореме 3 матрица $A$ - не фробениусова типа.

Норма построенной матрицы равна 42, о существовании матрицы не фробениусова типа с меньшей нормой автору ничего неизвестно.

\section{СПИСОК ЛИТЕРАТУРЫ}

[1] В.И. Арнольд. Цепные дроби. М.: МЦНМО, 2002. [2] Е.И. Коркина // Труды МИАН. 1995. Т. 209. С. 143-166.

Московский государственный Принято редколлегией университет им. М. В. Ломоносова 23.07.2004 